ఠ

\title{
A Case of Darier's Disease with a Novel Missense Mutation in ATP2A2 Successfully Treated with Calcipotriol/Betamethasone Dipropionate Two- Compound Ointment
}

\author{
Teppei Hagino $\mathbb{D}^{\prime}$, Hajime Nakano², Hidehisa Saeki $\mathbb{D}^{3}$, Naoko Kanda $\mathbb{D}^{\prime}$ \\ 'Department of Dermatology, Nippon Medical School Chiba Hokusoh Hospital, Inzai, Japan; ${ }^{2}$ Department of Dermatology, Hirosaki University \\ Graduate School of Medicine, Hirosaki, Japan; ${ }^{3}$ Department of Dermatology, Nippon Medical School, Bunkyo-Ku, Tokyo, Japan
}

Correspondence: Teppei Hagino, Department of Dermatology, Nippon Medical School Chiba Hokusoh Hospital, I7I5 Kamagari, Inzai, Chiba, 270-I694, Japan, Tel +8I 47699 I I I I, Fax +8I 47699 I9II, Email teppei-hagino@nms.ac.jp

\begin{abstract}
Darier's disease (DD) is a rare autosomal dominant genodermatosis caused by mutations in the ATP2A2 gene, which encodes for the sarcoendoplasmic reticulum calcium ATPase type 2 isoform (SERCA2). In epidermal keratinocytes, the decrease in SERCA2 inhibits the transportation of desmosomal proteins to the plasma membrane, resulting in acantholysis and dyskeratosis. We present the first case of DD with a novel missense mutation in the ATP2A2 gene and successfully treated with calcipotriol/ betamethasone dipropionate two-compound ointment. A 34-year-old Japanese woman presented with erythema and scales on the scalp and clusters of keratotic papules on the neck and groin. Similar symptoms were observed in her father, younger sister, and daughter. Histopathological examination revealed corps ronds in the granular layer and grains in the horny layer of the epidermis and acantholytic lacuna just above the basal layer. She was diagnosed with DD. A novel heterozygous missense mutation, c.616A $>$ C ( $\mathrm{p}$. Asn206His), was detected in the ATP2A2 gene in both the patient and her daughter. The patient was treated with calcipotriol/ betamethasone dipropionate two-compound ointment, which resulted in improvement of the skin eruption. This two-compound topical ointment may be a promising therapeutic strategy in the treatment for DD.
\end{abstract}

Keywords: sarcoendoplasmic reticulum calcium ATPase type 2 isoform, corticosteroid, 1, 25-dihydroxyvitamin D3, p.Asn206His

\section{Introduction}

Darier's disease (DD) is a rare autosomal dominant genodermatosis characterized by multiple firm, greasy, and hyperkeratotic papules that coalesce into plaques, and it mainly affects the seborrheic regions. ${ }^{1}$ Darier's disease is caused by a heterozygous mutation in the ATP2A2 gene on chromosome 12q23-24.1. ${ }^{2}$ The ATP2A2 gene encodes for the sarcoendoplasmic reticulum calcium ATPase type 2 isoform (SERCA2). ${ }^{3}$ The SERCA2 is a single-subunit integral membrane P-type ATPase that mediates the ATP-driven transport of cytosolic $\mathrm{Ca}^{2+}$ against a concentration gradient into the $\mathrm{Ca}^{2+}$ releasable store in the lumen of the endoplasmic reticulum (ER), and it plays an important role in intracellular $\mathrm{Ca}^{2+}$ signaling in keratinocytes. ${ }^{4}$ The malfunction or decrease in SERCA2 prevents cell-to-cell adhesion and differentiation of epidermal keratinocytes. ${ }^{4}$ There are no proven curative treatments for DD. A calcipotriol/betamethasone dipropionate two-compound ointment (Dovobet) is used to treat psoriasis vulgaris. ${ }^{5}$ Herein we present a case of DD with a novel missense mutation in $A T P 2 A 2$ successfully treated with the calcipotriol/betamethasone dipropionate two-compound ointment.

\section{Case Presentation}

A 34-year-old Japanese woman with 10 years of recurrent erythema and scales on the scalp and brownish keratotic papules on the neck and bilateral groin was referred to our department (Figure 1A and B). The patient was previously treated with topical 

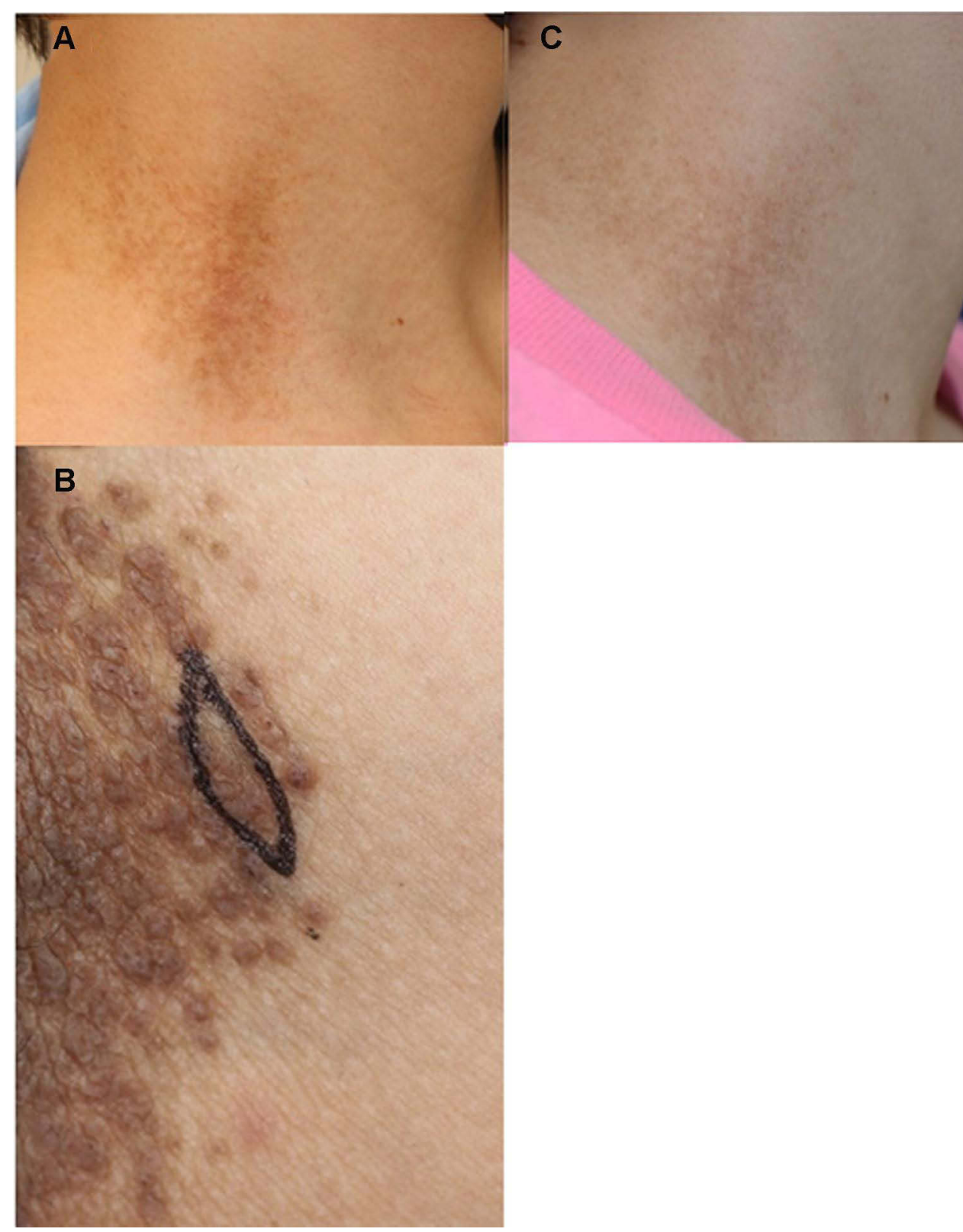

Figure I Brown keratotic papules on the neck (A), and a cluster of papules on the groin (B). The improvement of the eruption on the neck after 3 months of treatment with calcipotriol/betamethasone dipropionate two-compound ointment (C).

steroids, but the treatment efficacy was inconsistent. Similar eruptions were observed in her father, younger sister, and daughter. Pathological examination of the inguinal papules revealed dyskeratotic cells, such as corps ronds in the granular cell layer and grains in the horny layer, and acantholytic lacuna just above the basal layer (Figure 2). She was diagnosed with DD.

After obtaining informed consent from the patient, genomic DNA was extracted from her peripheral blood leukocytes, and all of the exons of the $A T P 2 A 2$ gene, including the intron-exon boundaries, were amplified by polymerase chain reaction. The protocol was conducted in accordance with the principles of the Declaration of Helsinki and was approved by the Hirosaki University Genetic Ethics Committee. Direct sequencing of $A T P 2 A 2$ detected a heterozygous missense mutation in exon 7, c.616A>C (p.Asn206His) (Figure 3), which was neither reported nor found in ethnically matched controls. The same mutation was observed in the daughter of the patient.

Although DD is known to respond well to oral etretinate therapy, ${ }^{1}$ this treatment has teratogenic side effects and is not appropriate for patients of a reproductive age. Thus, we selected a once-daily treatment with topical calcipotriol 

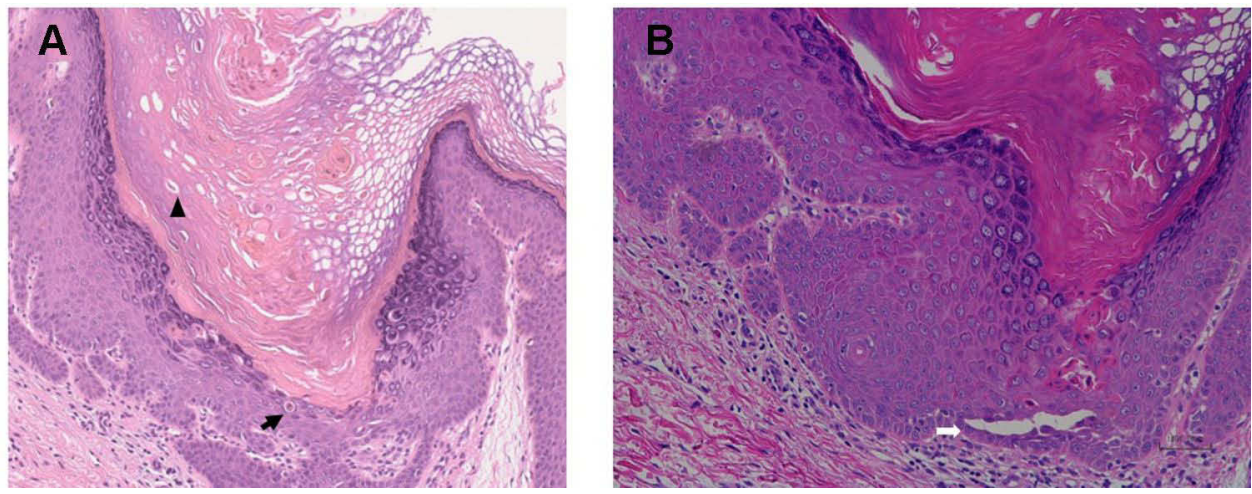

Figure 2 Histopathology showing dyskeratotic keratinocytes, including corps ronds in the granular layer (arrow) and grains in the horny layer (arrow, head) (A), and acantholytic lacuna just above the basal layer (white arrow) (B). Hematoxylin-eosin staining, $\times 100$.

(1,25-dihydroxyvitamin D3 analog)/betamethasone dipropionate (corticosteroid) two-compound ointment (LEO Pharma K.K., Chiyoda-ku, Tokyo, Japan). After 3 months of treatment, the erythema and scales on the scalp were reduced, and the papules on the neck and groin were flattened and decreased with mild residual pigmentation (Figure 1C).

\section{Discussion}

Darier's disease is a relatively rare autosomal dominant genodermatosis, with a prevalence of 1 in 30,000 to 100,000 people and no gender difference. ${ }^{1,6}$ In addition to the manifestation of hyperkeratotic papules in seborrheic or intertriginous areas, DD manifests with warty papules on the dorsum of hands and feet, palmoplantar pits, and longitudinal red or white stripes in the fingernails. ${ }^{6}$ These findings were not presented by the present case.

The present case demonstrates a novel missense mutation in exon 7 of the $A T P 2 A 2$ gene, c.616A $>\mathrm{C}$ (p.Asn206His), which was also inherited by her daughter. The $\mathrm{Ca}^{2+}$ pump SERCA2 encoded by $A T P 2 A 2$ is composed of 11 transmembrane helices harboring $\mathrm{Ca}^{2+}$ binding sites and a large cytosolic head consisting of actuator, nucleotide-binding, and phosphorylation domains. ${ }^{4}$ The patient's mutation resides in the actuator domain facing the cytoplasm and might impair the $\mathrm{Ca}^{2+}$ uptake and/or ATPase activities of SERCA2. ${ }^{4}$ The autosomal dominant inheritance of DD is mediated by haploinsufficiency, wherein one copy of the $A T P 2 A 2$ gene is inactivated or deleted, and the remaining functional copy of the gene is not adequate to sufficiently produce the gene product, SERCA2, which is required to preserve normal function. ${ }^{7}$ Certain ER stresses, such as wounds, infections, or UV exposure, to the keratinocytes of patients with DD may downregulate ATP2A2 expression from the intact allele, leading to the reduction in SERCA2 proteins and resultant depletion of the ER $\mathrm{Ca}^{2+}$ store. ${ }^{8}$ The depletion of the $\mathrm{ER} \mathrm{Ca}^{2+}$ store leads to the decreased expression of sphingosine kinase and its product, sphingosine 1-phosphate, ${ }^{9}$ and/or decreased activity of protein kinase C $\alpha$ in the ER. ${ }^{10}$ The suppression of these kinases impairs the distribution of desmoplakin, desmoglein 3, desmocollin 3, or E-cadherin to plasma membranes, inducing acantholysis, ${ }^{9,10}$ or reduces late-differentiation proteins in keratinocytes, such as involucrin, ${ }^{9}$ or survival proteins, such as Bcl- $2,{ }^{11}$ inducing abnormal differentiation and apoptosis, leading to dyskeratosis. ${ }^{9}$

Darier's disease is an intractable disease for which there are no proven curative treatments. ${ }^{1}$ It was recently reported that a low dose $(1-5 \mathrm{mg} / \mathrm{d})$ of opioid receptor antagonist naltrexone improved the mild-to-moderate forms of the clinical manifestation in patients with $\mathrm{DD}^{12}$ and may become a new treatment option by possibly affecting inflammation, desmosomal adhesion, or migration of keratinocytes. ${ }^{13}$ According to the comprehensive review of the literature on therapeutic options for $\mathrm{DD},{ }^{14}$ there was grade $\mathrm{B}$ evidence (weak recommendation; limited-quality, patient-oriented evidence) supporting the efficacy of topical 1,25-dihydroxyvitamin D3 analogs, whereas there was grade C evidence (weak recommendation; low-quality evidence) for the use of fluocinonide, a corticosteroid. Corticosteroids have been reported to be useful in $33 \%$ of DD patients, not all patients. ${ }^{15}$

The two-compound ointment administered in this study contains $50 \mu \mathrm{g} / \mathrm{g}$ calcipotriol (1,25-dihydroxyvitamin D3 analog) and $0.5 \mathrm{mg} / \mathrm{g}$ betamethasone dipropionate (corticosteroid). ${ }^{5}$ This is the first report demonstrating the therapeutic 

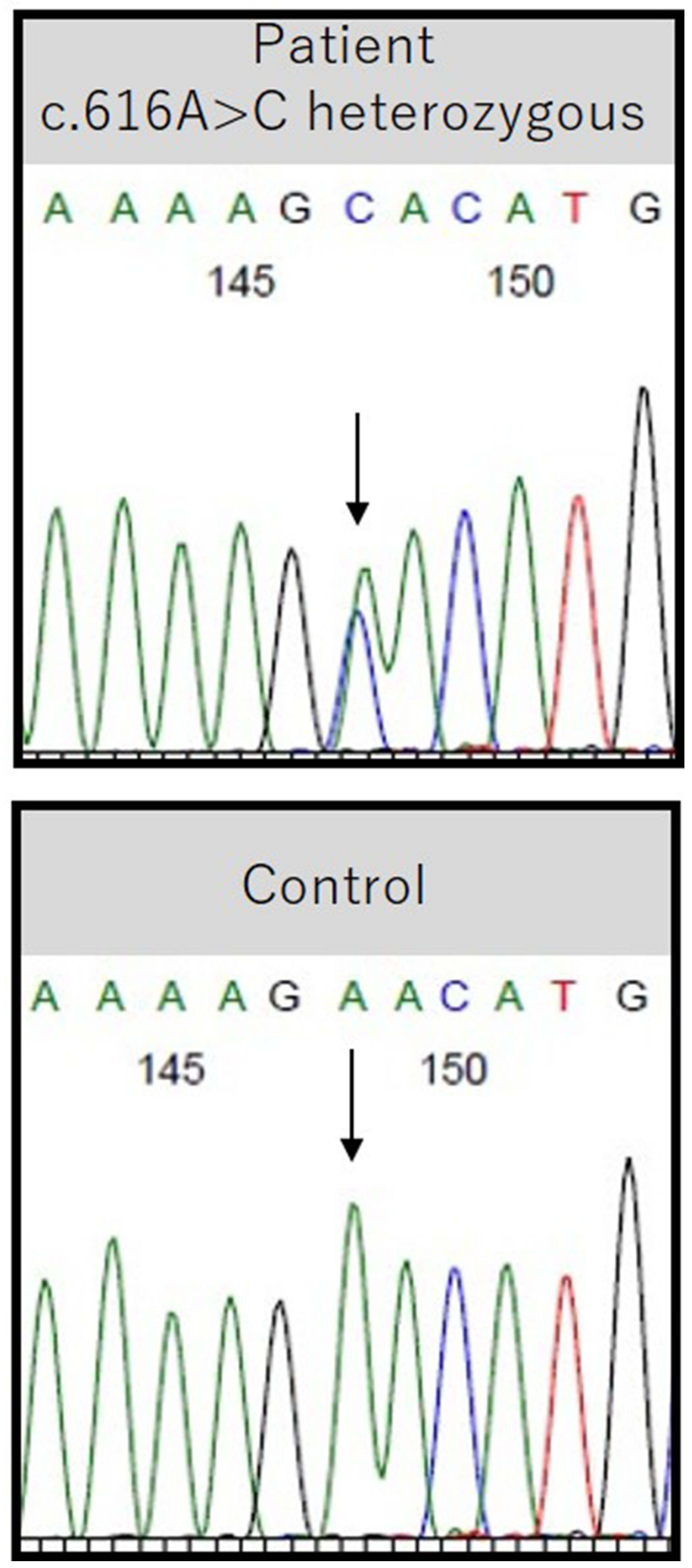

Figure 3 A heterozygous missense mutation in exon 7 of ATP2A2, c.616A>C (p.Asn206His), was detected in the patient. The reference sequence in the healthy controls is shown below. 
efficacy of this two-compound ointment for DD. The direct effects of corticosteroids on $\mathrm{Ca}^{2+}$ homeostasis in keratinocytes have not been reported, although several studies have reported that corticosteroids decrease or increase the intracellular $\mathrm{Ca}^{2+}$ concentration depending on the type of corticosteroid, concentration, and cell type. ${ }^{16}$ The corticosteroids suppressed the UVB-induced downregulation of $A T P 2 A 2 \mathrm{mRNA}$ levels in normal human keratinocytes in vitro, possibly via inhibition of UVB-induced interleukin- 6 production in these cells, ${ }^{17,18}$ indicating that corticosteroidmediated restoration occurred from the decrease in SERCA2 induced by ER stress.

It has been reported that 1,25-dihydroxyvitamin D3 activates phospholipase $\mathrm{C}$ in keratinocytes and generates inositol triphosphate and diacylglycerol; inositol triphosphate induces $\mathrm{Ca}^{2+}$ release from intracellular $\mathrm{Ca}^{2+}$ stores, and the intracellular $\mathrm{Ca}^{2+}$ further opens $\mathrm{Ca}^{2+}$ channels through which $\mathrm{Ca}^{2+}$ enters the keratinocytes. ${ }^{19,20}$ The $1,25-$ dihydroxyvitamin $\mathrm{D} 3$-induced $\mathrm{Ca}^{2+}$ signal and diacylglycerol promote the activity of protein kinase $\mathrm{C}$, which further enhances the expression of late-differentiation proteins in keratinocytes, such as involucrin, ${ }^{17}$ and stimulates the assembly of adherens junction proteins in keratinocytes. ${ }^{21}$ Furthermore, 1,25-dihydroxyvitamin D3 protects human keratinocytes from apoptosis by increasing the activity of sphingosine kinase. ${ }^{22}$ These reports indicate that 1,25 dihydroxyvitamin D3 may rescue keratinocytes from dyskeratosis and acantholysis induced by ER stress. These effects of corticosteroids and 1,25-dihydroxyvitamin D3 may additively or synergistically contribute to the therapeutic efficacy of the topical calcipotriol/betamethasone dipropionate two-compound product in the present case with DD. It has been reported that emollients improved symptoms, to some extent, in $29 \%$ of patients with DD. ${ }^{15}$ Since calcipotriol/ betamethasone dipropionate two-compound ointment contains white petrolatum, paraffin, and polyoxypropylene stearyl ether, these vehicles may have improved the skin appearance in the present case, and this possibility should be further examined. Short-term (less than 6 months) or intermittent use of the calcipotriol/betamethasone dipropionate twocompound ointment is recommended for DD patients since calcipotriol ointment may cause skin irritation, ${ }^{23}$ and longterm use of corticosteroid ointment may cause skin atrophy or telangiectasia. ${ }^{24}$

\section{Conclusion}

In conclusion, this is the first case of DD with a novel missense mutation in the $A T P 2 A 2$ gene, c.616A $>C$ ( $p$. Asn206His), which was successfully treated with a calcipotriol/betamethasone dipropionate two-compound ointment. Future studies should clarify the precise mechanisms by which this mutation causes skin eruption of DD and how topical corticosteroids and 1,25-dihydroxyvitamin D3 analogs additively or synergistically improve the symptoms of patients with DD.

\section{Ethics Statement}

Informed consent for publication of the case report and associated images was obtained from the patient.

\section{Disclosure}

Professor Hidehisa Saeki reports grants and/or personal fees from Mitsubishi Tanabe Pharma Corporation, Taiho Pharmaceutical Co., Ltd., Torii Pharmaceutical Co. Ltd., Maruho Co., Ltd., Kyowa Kirin Co., Ltd., Sanofi K.K., AbbVie GK, Novartis Pharma K.K., Eli Lilly Japan K.K., KYORIN Pharmaceutical Co., Ltd., Eisai Co., Ltd., Tokiwa Pharmaceutical Co., Ltd., Japan Tobacco Inc., and LEO Pharma K.K., outside the submitted work. The authors report no other conflicts of interest in this work.

\section{References}

1. Haber RN, Dib NG. Management of Darier disease: a review of the literature and update. Indian J Dermatol Venereol Leprol. 2021;87:14-21. doi:10.25259/IJDVL_963_19

2. Bashir R, Munro CS, Mason S, Stephenson A, Rees JL, Strachan T. Localisation of a gene for Darier's disease. Hum Mol Genet. 1993;2:1937-1939. doi:10.1093/hmg/2.11.1937

3. Sakuntabhai A, Ruiz-Perez V, Carter S, et al. Mutations in ATP2A2, encoding a Ca2+ pump, cause Darier disease. Nat Genet. 1999;21:271-277. doi: $10.1038 / 6784$

4. Dode L, Andersen JP, Leslie N, Dhitavat J, Vilsen B, Hovnanian A. Dissection of the functional differences between sarco (endo) plasmic reticulum Ca2+-ATPase (SERCA) 1 and 2 isoforms and characterization of Darier disease (SERCA2) mutants by steady-state and transient kinetic analyses. J Biol Chem. 2003;278:47877-47889. doi:10.1074/jbc.M306784200 
5. van de Kerkhof PC. The impact of a two-compound product containing calcipotriol and betamethasone dipropionate (Daivobet/ Dovobet) on the quality of life in patients with psoriasis vulgaris: a randomized controlled trial. Br J Dermatol. 2004;151:663-668. doi:10.1111/j.13652133.2004.06134.x

6. Onozuka T, Sawamura D, Yokota K, Shimizu H. Mutational analysis of the ATP2A2 gene in two Darier disease families with intrafamilial variability. Br J Dermatol. 2004;150:652-657. doi:10.1111/j.0007-0963.2004.05868.x

7. Cooper SM, Burge SM. Darier's disease: epidemiology, pathophysiology, and management. Am J Clin Dermatol. 2003;4:97-105. doi:10.2165/ 00128071-200304020-00003

8. Seo SH, Kim SE, Lee SE. ER stress induced by ER calcium depletion and UVB irradiation regulates tight junction barrier integrity in human keratinocytes. J Dermatol Sci. 2020;98:41-49. doi:10.1016/j.jdermsci.2020.02.006

9. Celli A, Mackenzie DS, Zhai Y, et al. SERCA2-controlled $\mathrm{Ca}^{2}+$-dependent keratinocyte adhesion and differentiation is mediated via the sphingolipid pathway: a therapeutic target for Darier's disease. J Invest Dermatol. 2012;132:1188-1195. doi:10.1038/jid.2011.447

10. Hobbs RP, Amargo EV, Somasundaram A, et al. The calcium ATPase SERCA2 regulates desmoplakin dynamics and intercellular adhesive strength through modulation of PKC $\alpha$. FASEB J. 2011;25:990-1001. doi:10.1096/fj.10-163261

11. Pasmatzi E, Badavanis G, Monastirli A, Tsambaos D. Reduced expression of the antiapoptotic proteins of Bcl-2 gene family in the lesional epidermis of patients with Darier's disease. J Cutan Pathol. 2007;34:234-238. doi:10.1111/j.1600-0560.2006.00600.x

12. Boehmer D, Eyerich K, Darsow U, Biedermann T, Zink A. Variable response to low-dose naltrexone in patients with Darier disease: a case series. J Eur Acad Dermatol Venereol. 2019;33:950-953. doi:10.1111/jdv.15457

13. Lee B, Elston DM. The uses of naltrexone in dermatologic conditions. J Am Acad Dermatol. 2019;80:1746-1752. doi:10.1016/j.jaad.2018.12.031

14. Hanna N, Lam M, Fleming P, Lynde C. Therapeutic options for the treatment of Darier's disease: a comprehensive review of the literature. $J$ Cutan Med Surg. 2021;12034754211058405. doi:10.1177/12034754211058405

15. Burge SM, Wilkinson JD. Darier-White disease: a review of the clinical features in 163 patients. J Am Acad Dermatol. 1992;27:40-50. doi:10.1016/0190-9622(92)70154-8

16. Suwanjang W, Holmström KM, Chetsawang B, Abramov AY. Glucocorticoids reduce intracellular calcium concentration and protects neurons against glutamate toxicity. Cell Calcium. 2013;53:256-263. doi:10.1016/j.ceca.2012.12.006

17. Mayuzumi N, Ikeda S, Kawada H, Ogawa H. Effects of drugs and anticytokine antibodies on expression of ATP2A2 and ATP2C1 in cultured normal human keratinocytes. Br J Dermatol. 2005;152:920-924. doi:10.1111/j.1365-2133.2005.06394.x

18. Kirnbauer R, Köck A, Neuner P, et al. Regulation of epidermal cell interleukin-6 production by UV light and corticosteroids. J Invest Dermatol. 1991;96:484-489. doi:10.1111/1523-1747.ep12470181

19. Bikle DD. 1,25(OH)2D3-modulated calcium induced keratinocyte differentiation. J Investig Dermatol Symp Proc. 1996;1:22-27.

20. Tang W, Ziboh VA. Agonist/inositol trisphosphate-induced release of calcium from murine keratinocytes: a possible link with keratinocyte differentiation. J Invest Dermatol. 1991;96:134-138. doi:10.1111/1523-1747.ep12515934

21. Gniadecki R, Gajkowska B, Hansen M. 1,25-dihydroxyvitamin D3 stimulates the assembly of adherens junctions in keratinocytes: involvement of protein kinase C. Endocrinology. 1997;138:2241-2248. doi:10.1210/endo.138.6.5156

22. Manggau M, Kim DS, Ruwisch L, et al. 1alpha,25-dihydroxyvitamin D3 protects human keratinocytes from apoptosis by the formation of sphingosine-1-phosphate. J Invest Dermatol. 2001;117:1241-1249. doi:10.1046/j.0022-202x.2001.01496.x

23. Kragballe K, Steijlen PM, Ibsen HH, et al. Efficacy, tolerability, and safety of calcipotriol ointment in disorders of keratinization. Results of a randomized, double-blind, vehicle-controlled, right/left comparative study. Arch Dermatol. 1995;131:556-560. doi:10.1001/archderm.1995.01690170058008

24. Mills CM, Marks R. Side effects of topical glucocorticoids. Curr Probl Dermatol. 1993;21:122-131.

Clinical, Cosmetic and Investigational Dermatology is an international, peer-reviewed, open access, online journal that focuses on the latest clinical and experimental research in all aspects of skin disease and cosmetic interventions. This journal is indexed on CAS. The manuscript management system is completely online and includes a very quick and fair peer-review system, which is all easy to use. Visit http://www. dovepress.com/testimonials.php to read real quotes from published authors.

Submit your manuscript here: https://www.dovepress.com/clinical-cosmetic-and-investigational-dermatology-journal 